\title{
Neue Leitlinie zu Weichgewebesarkomen verabschiedet
}

Zu der Bedeutung der neuen Leitlinie befragte die Röfo Prof. Dr. Rolf Janka als Mandatsträger der DRG und Prof. Dr. Andreas Mahnken als Mandatsträger der DeGIR.

Was war die Herausforderung der S3-Leitlinie Adulte Weichteilsarkome?

Janka: Die S3-Leitlinie Adulte Weichteilsarkome ist als Gesamtwerk neu, es gab also keine Blaupause, an der man sich orientieren konnte. Weichteilsarkome sind eine heterogene Gruppe von Tumoren und sämtliche Entitäten kommen selten vor. Somit ist die Datenlage eher spärlich, wodurch die Expertenmeinung an Stellenwert gewinnt.

Mahnken: Im deutschsprachigen Raum gibt es zwar bisher Leitlinien zu einzelnen Aspekten der Weichgewebssarkome, beispielsweise

\section{Leitliniensteckbrief}

\begin{tabular}{|l|l}
\hline Name der LL: & Adulte Weichgewebesarkome \\
\hline $\begin{array}{l}\text { Zielorientierung } \\
\text { der Leitlinie: }\end{array}$ & $\begin{array}{l}\text { Empfehlungen für eine evidenzbasierte Diagnostik und Therapie } \\
\text { in Abhängigkeit von Histologie und Tumorstadium }\end{array}$ \\
\hline Klassifikation: & $\mathrm{S} 3$ \\
\hline Registernummer & $032-044 \mathrm{OL}$ \\
\hline $\begin{array}{l}\text { Federführende } \\
\text { Fachgesellschaft: }\end{array}$ & Deutsche Krebsgesellschaft e. V. (DKG) \\
\hline $\begin{array}{l}\text { Mandatsträger aus der } \\
\text { Radiologie: }\end{array}$ & $\begin{array}{l}\text { Prof. Dr. Rolf Janka, Prof. Dr. Karl-Friedrich Kreitner, } \\
\text { Prof. Dr. Andreas Mahnken }\end{array}$ \\
\hline
\end{tabular}

in der Gynäkologie, aber ein zusammenhängendes Werk fehlte bisher. Die Erstellung dieser Leitlinie war aus interventioneller Sicht besonders herausfordernd, da die Datenlage zu interventionellen Verfahren sehr schlecht ist. Hier existieren lediglich Kasuistiken und Kohortenstudien niedrigen Evidenzlevels.
Selbst die Datenlage zur Indikation und Durchführung der perkutanen Biopsie versus offene Biopsie ist heterogen und entsprechend kontrovers diskutiert. 


\section{Was sind die Kerninhalte der Leitlinie aus Sicht der Radiologie?}

Janka: Die Planung der Therapie von Weichteilsarkomen wird in einem Tumorboard mit radiologischer Beteiligung festgelegt. Die Bildgebung bei Verdacht auf Sarkom und bei gesichertem Sarkom beruht auf Ultraschall und MRT. Dabei wird in der Leitlinie sehr genau beschrieben, wie die MRT durchgeführt werden sollte. Kernpunkte sind die KM-Gabe und die Diffusionswichtung, um die Regionen des Tumors mit der höchsten Entdifferenzierung zu detektieren.

Für die Artdiagnose des Tumors wird in der Leitlinie die bildgeführte und damit in erster Linie radiologische Stanzbiopsie genannt.
Mahnken: In der Therapie können nach interdisziplinärem Konsil in besonderen Situationen auch interventionelle Verfahren wie Ablation und Embolisation Anwendung finden.

Janka: Bei der Nachsorge hat die CT des Thorax, neben dem Thorax-Röntgen, den Einzug in die Leitlinie geschafft. Obwohl die Datenlage keinen klaren Vorteil für die CT-Thorax zeigt, wird sie als „bevorzugte Alternative zur konventionellen Röntgenuntersuchung“ genannt.

Wie ändert sich durch die Leitlinie die radiologische Versorgung der Patientinnen und Patienten?

Mahnken: Die Indikation und Anwendung der perkutanen Biopsie im Rahmen der Diagnose- findung wurde klar und modern definiert. Dies bietet für den Patienten die Sicherheit einer homogenen Versorgungsqualität. Der Stellenwert interventioneller Verfahren wurde erstmals in einer Leitlinie festgeschrieben und bietet geeigneten Patienten damit die Möglichkeit schonender Therapieoptionen.

Janka: Der diagnostisch-radiologische Teil der Leitlinie ist sehr konkret und modern. Für die Patienten sollte die Leitlinie zu einer zunehmenden Standardisierung der MRProtokolle und damit zu einer besseren Vergleichbarkeit der Untersuchungen von unterschiedlichen MR-Zentren führen. Hilfreich hierfür wäre ein konkreter Protokollvorschlag durch die AG MSK, der bereits in Arbeit ist. 